\title{
Adaptive Filtering for Stochastic Systems With Generalized Disturbance Inputs
}

\author{
Yan Liang, Member, IEEE, Donghua Zhou, Senior Member, IEEE, Lei Zhang, Member, IEEE, and
} Quan Pan, Member, IEEE

\begin{abstract}
This letter presents a new class of discrete-time linear stochastic systems with the statistically-constrained disturbance input, which can represent an arbitrary linear combination of dynamic, random, and deterministic disturbance inputs to generalize the complicated modeling error encountered in actual applications. An adaptive filtering scheme is proposed for such systems by recursively constructing and adaptively minimizing the upper-bounds of covariance matrices of the state predictions, innovations, and estimates. The minimum-upper-bound filter is then obtained via online scalar convex optimization. The experiment on maneuvering target tracking shows that the proposed filter can significantly reduce the peak estimation errors due to maneuvers, compared with the well-known IMM method.
\end{abstract}

Index Terms-Adaptive Kalman filtering, discrete time systems, stochastic systems.

\section{INTRODUCTION}

A $\mathrm{S}$ an optimal linear minimum mean square error estimator, the Kalman filter (KF) is widely used in signal processing. However, its performance will degrade greatly if the modeling errors caused by parameter variations and external disturbances cannot be well represented. It has motivated many studies of adaptive filtering in the presence of disturbance inputs (DI) to the system model of KF.

Many strategies were proposed to model DI. First, it is modeled as zero-mean random noise with unknown covariance. The filter design is based on the online covariance identification via Bayesian or maximum likelihood estimation for stationary noise process in linear time-invariant systems [1], [2]. Recently, filter design was extended to time-variant covariance [3] and jump Markov stochastic systems [4]. Second, the DI is assumed to be deterministic. Using least-square estimation and moving-window hypothesis testing, the filters can deal with the DI which is piecewise-constant [5] or a sum of basis functions with piecewise-constant weights [6]. Third, the DI is arbitrary but the rank of its distribution matrix is less than that of measurement matrix. The corresponding filter is an

Manuscript received April 12, 2008; revised June 09, 2008. This work was supported in part by the Natural Science Foundation of China (NSFC) under Grant no. 60634030, 60721003, and 60736026 and in part by the Program for New Century Talents in Chinese University. The associate editor coordinating the review of this manuscript and approving it for publication was Prof. Behrouz Farhang-Boroujeny.

Y. Liang and Q. Pan are with the College of Automation, Northwestern Polytechnical University, Xi'an, China (e-mail: liangyan@nwpu.edu.cn; quanpan@nwpu.edu.cn).

D. H. Zhou is with the Department of Automation, Tsinghua University, Beijing 100081, China (e-mail: zdh@mail.tsinghua.edu.cn).

L. Zhang is with the Department of Computing, The Hong Kong Polytechnic University, Hong Kong (e-mail: cslzhang@comp.polyu.edu.hk).

Digital Object Identifier 10.1109/LSP.2008.2002707 asymptotically-stable and DI-decoupling observer [7]. Fourth, the DI is norm-bounded and the robust filters are designed offline to minimize the gain of the transfer function from DI to the estimation error [8]. Fifth, the DI is a randomly switching parameter obeying a known Markov chain. The filters are multiple model estimators [9]-[13], such as the interacting multiple model (IMM) which is well-known in maneuvering target tracking.

In general, the above filters are DI-specific due to their significant differences in both DIs and solutions. The actual applications, however, encounter much more complicated DIs. It is highly demanded to generalize the DIs and pursue the corresponding filter. To the best of our knowledge, little research has been reported on this topic.

This letter presents a generalized type of DI - a class of statistically constrained DIs, which can represent an arbitrary linear combination of dynamic DIs, random DIs, and deterministic DIs. The proposed filter can adaptively minimize the upper bounds of covariance matrices of the state prediction, filtering residual, and state estimate.

Throughout this letter, for any two square matrices, $\mathrm{A}$ and $\mathrm{B}, \mathrm{A}-\mathrm{B} \geq 0$ and $\mathrm{A}-\mathrm{B}>0$ mean that $\mathrm{A}-\mathrm{B}$ is positive semi-definite and positive definite, respectively. Symbol "=:" means definition.

\section{PROBLEM FORMULATION}

Consider a new discrete-time linear stochastic system

$$
\left\{\begin{array}{l}
x_{k+1}=F_{k} x_{k}+B_{k} u_{k}+\Gamma_{k} q_{k}+\delta_{k} \\
y_{k+1}=H_{k+1} x_{k+1}+v_{k+1}
\end{array}\right.
$$

where $x \in R^{n}, u \in R^{l}$, and $y \in R^{m}$ represent the system state, control input, and measurement, respectively. The matrices $F$, $B, \Gamma$, and $H$ are known with proper dimension. The process noise $q \in R^{p}$ and measurement noise $v \in R^{m}$ are zero-mean white noises with known covariance $Q_{k} \geq 0$ and $R_{k+1}>0$, respectively. Noises $q$ and $v$ and the initial state $x_{0}$ are independent. The introduced new term $\delta_{k}$ satisfies

$$
\left\{\begin{array}{l}
E\left\{\delta_{k} q_{j}^{T}\right\}=\mathbf{O}_{n \times p} \\
E\left\{\delta_{k} \nu_{j+1}^{T}\right\}=\mathbf{O}_{n \times m}
\end{array} \quad(\forall j \geq k)\right.
$$

where $O_{n \times p}$ and $O_{n \times m}$ are zero matrices with dimension $n \times p$ and $n \times m$, respectively. Denote by $\bar{Q}^{k-1}=:\left[q_{0}^{T}, \ldots, q_{k-1}^{T}\right]^{T}$, $\bar{V}^{k}=:\left[v_{1}^{T}, \ldots, v_{k}^{T}\right]^{T}, \bar{\Delta}^{k-1}=:\left[\delta_{0}^{T} \cdots \delta_{k-1}^{T}\right]^{T}$, and $\bar{W}^{k}=:$ $\left[\omega_{0}^{T}, \ldots, \omega_{k}^{T}\right]^{T}$, where $\omega_{k}$ is a random noise which is uncorrelated with $q$ and $v$ but whose mean and covariance are unknown. Let $f_{1 k}$ be an arbitrary linear time-variant function of $\bar{Q}^{k-1}$, $\bar{V}^{k}$, and $\bar{\Delta}^{k-1}$; let $f_{2 k}$ be an arbitrary deterministic time-variant 
function; and let $f_{k}$ be an arbitrary linear weighted sum of $f_{1 k}$, $f_{2 k}$, and $\omega_{k}$.

1) Remark 2.1: Repeatedly substituting $f_{i}$ by $f_{i-1}(i \leq k)$, it is clear that $f_{k}$ is a linear function of $\bar{Q}^{k-1}, \bar{V}^{k}, \bar{W}^{k}$, and $\bar{\Delta}^{k-1}$. With the fact that $\bar{Q}^{k-1}, \bar{V}^{k}, \bar{W}^{k}$, and $\bar{\Delta}^{k-1}$ are linearly independent of $q_{j}$ and $v_{j+1}(\forall j \geq k)$, it is concluded that $f_{k}$ is linearly independent of $q_{j}$ and $v_{j+1}$, and thus, $\delta_{k}=f_{k}$ satisfies (2). Therefore, $\delta_{k}$ can represent an arbitrary linear weighted sum of $f_{1 k}, f_{2 k}$, and $\omega_{k}$ even the corresponding weight matrices are unknown. Here $f_{1 k}, f_{2 k}$, and $\omega_{k}$ represent a class of DIs with dynamic property, deterministic DIs, and random DIs, respectively. That is to say, $\delta_{k}$ in (1) is a type of generalized DI (GDI) to represent the complicated modeling error. Because the existing filters are DI-specific and cannot be extended to deal with $\delta_{k}$, the new filter is required.

\section{UPPER BOUND FILTER DESIGN}

1) Definition 3.1: A linear filter (3)-(5) for system (1)-(2) is called an upper bound filter (UBF)

$$
\begin{aligned}
\text { state prediction } \hat{x}_{k+1 \mid k} & =F_{k} \hat{x}_{k \mid k}+B_{k} u_{k} \\
\text { filtering residual } \gamma_{k+1} & =y_{k+1}-H_{k+1} \hat{x}_{k+1 \mid k} \\
\text { state estimate } \hat{x}_{k+1 \mid k+1} & =\hat{x}_{k+1 \mid k}+K_{k+1} \gamma_{k+1}
\end{aligned}
$$

if there exist a sequence of positive-definite matrices $P_{k+1 \mid k}^{*}$, $V_{k+1}^{*}$, and $P_{k+1 \mid k+1}^{*}$ that satisfy

$$
\begin{aligned}
P_{k+1 \mid k}^{*} & \geq P_{k+1 \mid k}=: E\left\{\widetilde{x}_{k+1 \mid k} \widetilde{x}_{k+1 \mid k}^{T}\right\} \\
V_{k+1}^{*} & \geq V_{k+1}=: E\left\{\gamma_{k+1} \gamma_{k+1}^{T}\right\} \\
P_{k+1 \mid k+1}^{*} & \geq P_{k+1 \mid k+1}=: E\left\{\widetilde{x}_{k+1 \mid k+1} \widetilde{x}_{k+1 \mid k+1}^{T}\right\}
\end{aligned}
$$

where

$$
\begin{aligned}
\text { state prediction error } \widetilde{x}_{k+1 \mid k} & =: x_{k+1}-\hat{x}_{k+1 \mid k} \\
\text { state estimate error } \widetilde{x}_{k \mid k} & =: x_{k}-\hat{x}_{k \mid k}
\end{aligned}
$$

and the filter gain $K_{k+1}$ is a function of $P_{k+1 \mid k}^{*}$ and $V_{k+1}^{*}$.

2) Remark 3.1: Here $\delta_{k}$ cannot be determined from the measurements up to time $k$ and thus cannot be compensated in state prediction. Thus, the expression of the state prediction and measurement prediction (3)-(4) is the same as those of KF with the nominal model [equivalently (1)-(2) with $\delta \equiv 0$ ]. Because (5) is suitable for any linear estimate, the filter (3)-(5) is general.

3) Remark 3.2: Putting (1), (3), and (10) into (9), the state prediction error is

$$
\widetilde{x}_{k+1 \mid k}=F_{k} \widetilde{x}_{k \mid k}+\delta_{k}+\Gamma_{k} q_{k} .
$$

From (2) and the fact that the zero-mean white noises $q$ and $v$ are independent, it is easy to testify the linear independence between $F_{k} \widetilde{x}_{k \mid k}+\delta_{k}$ and $q_{k}$ and the linear independence between $\widetilde{x}_{k+1 \mid k}$ and $v_{k+1}$. Thus, putting (11) into (6) will lead to (12); putting (1), (4), and (9) into (7) will lead to (13); and putting (5) and (8) into (10) will lead to (14) as follows:

$$
\begin{aligned}
P_{k+1 \mid k}= & E\left\{\left[F_{k} \widetilde{x}_{k \mid k}+\delta_{k}\right]\left[F_{k} \widetilde{x}_{k \mid k}+\delta_{k}\right]^{T}\right\}+\Gamma_{k} Q_{k} \Gamma_{k}^{T} \\
V_{k+1}= & H_{k+1} P_{k+1 \mid k} H_{k+1}^{T}+R_{k+1} \\
P_{k+1 \mid k+1}= & \left(I-K_{k+1} H_{k+1}\right) P_{k+1 \mid k}\left(I-K_{k+1} H_{k+1}\right)^{T} \\
& +K_{k+1} R_{k+1} K_{k+1}^{T} .
\end{aligned}
$$

The GDI $\delta_{k}$ in (12) is the barrier to filter implementation. It is not feasible to directly estimate $P_{k+1 \mid k}$ with $n \times(n+1) / 2$ independent parameters except for $n=1$ because the measurement at time $k$ can only supply at most $n$ independent equations for possible parameter estimation. This is based on the fact that the rank of the $m \times n$ measurement matrix is less than $n+1$. Therefore, it is infeasible to design the filter by estimating the covariance of system (1)-(2). The idea of the UBF design is motivated by the fact that determining the upper bound, instead of the covariance, requires fewer parameters to be estimated.

As shown in (12), $\delta_{k}$ appears in the covariance of the state prediction in UBFs, as the system uncertainty. Thus, we have

$$
\begin{aligned}
P_{k+1 \mid k} & \geq E\left\{F_{k} \widetilde{x}_{k \mid k} \widetilde{x}_{k \mid k}^{T} F_{k}^{T}\right\}+\Gamma_{k} Q_{k} \Gamma_{k}^{T} \\
& =F_{k} P_{k \mid k} F_{k}^{T}+\Gamma_{k} Q_{k} \Gamma_{k}^{T} .
\end{aligned}
$$

Meanwhile, $V_{k+1}$ and $P_{k+1 \mid k+1}$ have nothing to do with $\delta_{k}$ given $P_{k+1 \mid k}$. Hence, the following recursive upper-bound structure is considered:

$$
\begin{aligned}
P_{k+1 \mid k}^{*}= & \alpha_{k} F_{k} P_{k \mid k}^{*} F_{k}^{T}+\Gamma_{k} Q_{k} \Gamma_{k}^{T} \\
V_{k+1}^{*}= & H_{k+1} P_{k+1 \mid k}^{*} H_{k+1}^{T}+R_{k+1} \\
P_{k+1 \mid k+1}^{*}= & \left(I-K_{k+1} H_{k+1}\right) P_{k+1 \mid k}^{*}\left(I-K_{k+1} H_{k+1}\right)^{T} \\
& +K_{k+1} R_{k+1} K_{k+1}^{T}
\end{aligned}
$$

where the fading factor $\alpha_{k} \geq 1$ is a parameter to be estimated.

It is needed to know whether there exists a UBF based on (15)-(17). If a UBF exists, it is further needed to determine the optimal filter parameters through minimizing the upper bounds so that the minimum UBF (MUBF) can be obtained. The following theorem provides the solution.

4) Theorem 3.1: If the following three conditions are satisfied:

$$
\text { (i) } P_{0 \mid 0}^{*} \geq P_{0 \mid 0}
$$

(ii) $H_{k}$ is of full column rank, i.e.,

$$
\begin{gathered}
\operatorname{rank}\left\{H_{k}\right\}=n, \forall k \\
\quad \text { (iii) } V_{k+1}^{*} \geq V_{k+1}
\end{gathered}
$$

then there exists a UBF with structure (15)-(17) and optimal parameters $\alpha_{k}^{O p t}$ and $K_{k+1}^{O p t}$. For any $\alpha_{k}$ satisfying $V_{k+1} \leq$ $\left.V_{k+1}^{*}\right|_{\alpha_{k}}$ and any filter gain $K(k+1)$, there exist

$$
\begin{aligned}
P_{k+1 \mid k} & \leq\left. P_{k+1 \mid k}^{*}\right|_{\alpha_{k}^{O p t}} \leq\left. P_{k+1 \mid k}^{*}\right|_{\alpha_{k}} \\
V_{k+1} & \leq\left. V_{k+1}^{*}\right|_{\alpha_{k}^{O p t}} \leq\left. V_{k+1}^{*}\right|_{\alpha_{k}} \\
\left.P_{k+1 \mid k+1}\right|_{K_{k+1}} & \leq\left. P_{k+1 \mid k+1}^{*}\right|_{\alpha_{k}^{O p t}, K_{k+1}^{O p t}} \\
& \leq\left. P_{k+1 \mid k+1}^{*}\right|_{\alpha_{k}, K_{k+1}}
\end{aligned}
$$

and the optimal filter parameters are

$$
\begin{aligned}
\alpha_{k}^{O p t} & =\min \left\{\alpha_{k} \mid \alpha_{k} \in \Lambda_{k}\right\} \\
K_{k+1}^{O p t} & =P_{k+1 \mid k}^{*} H_{k+1}^{T} V_{k+1}^{*-1}
\end{aligned}
$$

where $\Lambda_{k}=:\left\{\alpha_{k}\left|\alpha_{k} \geq 1, V_{k+1}^{*}\right|_{\alpha_{k}} \geq V_{k+1}\right\}$.

Proof: See the Appendix . 
5) Remark 3.3: In the traditional $\mathrm{KF}, P_{0 \mid 0}$ is known a priori. In the UBF proposed in this letter, only its upper bound $P_{0 \mid 0}^{*}$ is needed. Constraint (19) requires that the current measurement of system (1)-(2) should provide enough information for disturbance compensation. The third condition of Theorem 3.1 means that the fading factor $\alpha_{k}$ should be large enough to guarantee the existence of (7).

Putting (13) and (16) into (24), the optimal fading factor is the solution to the following scalar convex optimization:

$$
\alpha_{k}^{O p t}=\min \alpha_{k}
$$

which is subject to

1) $\alpha_{k} \geq 1$

2) $\alpha_{k} \bar{H}_{k+1} F_{k} P_{k \mid k}^{*} F_{k}^{T} H_{k+1}^{T}+H_{k+1} \Gamma_{k} Q_{k} \Gamma_{k}^{T} H_{k+1}^{T}+R_{k+1} \geq$ $V_{k+1}$.

In (26), $V_{k+1}$ is unknown and thus substituted by its unbiased estimate $\hat{V}_{k+1}=: \gamma_{k+1} \gamma_{k+1}^{T}$. Then linear matrix inequality (LMI) [14] is used to solve (26). The MUBF is expected robust to such approximation due to its robustness to parameter inaccuracy in pursuing the best solution in the "worst" possible case.

The recursive MUBF algorithm is summarized as follows: compute $\hat{x}_{k+1 \mid k}$ by (3) and $\gamma_{k+1}$ by (4); then determine $\alpha_{k}^{\text {Opt }}$ via LMI optimization using (26), where $V_{k+1}$ is substituted by $\hat{V}_{k+1}$; compute $\left.P_{k+1 \mid k}^{*}\right|_{\alpha_{k}^{\text {Opt }}}$ by (15), $\left.V_{k+1}^{*}\right|_{\alpha_{k}^{\text {opt }}}$ by $(16), K_{k+1}^{O p t}$ by (25), $\hat{x}_{k+1 \mid k+1}$ by (5), and $\left.P_{k+1 \mid k+1}^{*}\right|_{\alpha_{k}^{O p t}, K_{k+1}^{O p t}}$ by (17).

\section{EXPERIMENT}

The experiment of maneuvering target tracking in the benchmark target tracking scenario [10] was performed to evaluate the proposed MUBF in comparison with the well-known IMM [9]. The position, velocity, and acceleration of a maneuvering target in a two-dimensional $\xi-\eta$ plane are shown in Fig. 1. The sampling period is $1 \mathrm{~s}$. The observation is

$$
z_{k+1}=\left[\begin{array}{c}
\sqrt{\xi_{k+1}^{2}+\eta_{k+1}^{2}} \\
\arctan \left(\frac{\eta_{k+1}}{\xi_{k+1}}\right)
\end{array}\right]+\nu_{k+1}
$$

where $\nu_{k+1}$ is zero-mean Gaussian measurement noise with covariance $R_{k+1}=\operatorname{diag}\left\{\sigma_{1}{ }^{2}, \sigma_{2}{ }^{2}\right\}$. Through the first-order linearization of (27), as in the extended Kalman filter (EKF), the parameters of measurement equation in (1) are

$$
\begin{gathered}
y_{k+1}=z_{k+1}-\left[\begin{array}{c}
\sqrt{\hat{\xi}_{k+1 \mid k}^{2}+\hat{\eta}_{k+1 \mid k}^{2}} \\
\arctan \left(\frac{\hat{\eta}_{k+1 \mid k}}{\hat{\xi}_{k+1 \mid k}}\right)
\end{array}\right] \\
H_{k+1}=\left.\left[\frac{\left.\frac{\partial\left(\sqrt{\xi_{k+1}^{2}+\eta_{k+1}^{2}}\right)}{\partial x^{T}}\right]}{\partial\left(\arctan \left(\frac{\eta_{k+1}}{\xi_{k+1}}\right)\right)}\right]\right|_{x=\hat{x}_{k+1 \mid k}} ^{\partial x^{T}} .
\end{gathered}
$$

Here the design of IMM is the same as that in [10]. The proposed MUBF treats the unknown maneuver changes in velocity as GDIs to a nominal constant-velocity model. In MUBF design, the state equation in (1) has $x=[\xi \dot{\xi} \eta \dot{\eta}]^{T}, F=$ $\operatorname{diag}\left\{\left[\begin{array}{ll}1 & 1 \\ 0 & 1\end{array}\right],\left[\begin{array}{ll}1 & 1 \\ 0 & 1\end{array}\right]\right\} . B_{k} u_{k}$ and $\Gamma_{k} q_{k}$ are $4 \times 1$ zero vectors and $\delta_{k}=x_{k+1}-F_{k} x_{k}$. It should be stressed that the process noise is set to be zero in the MUBF. That is to say, unlike in
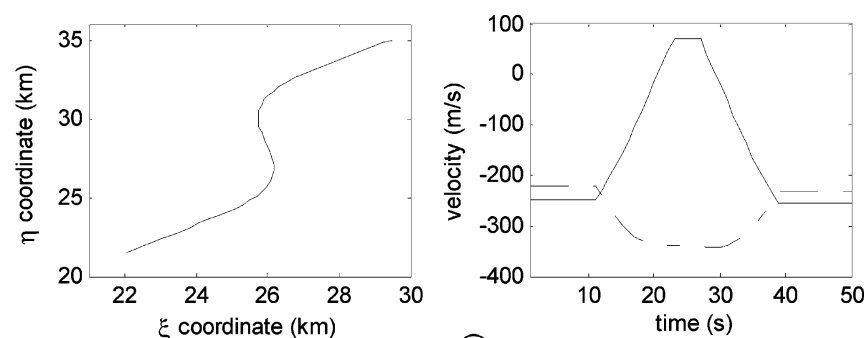

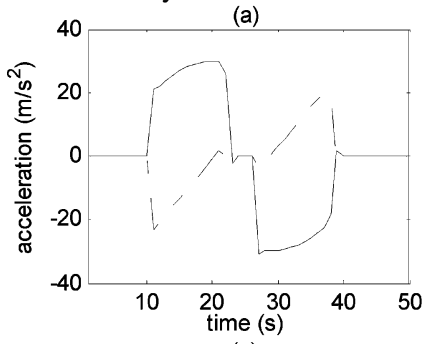

(c)

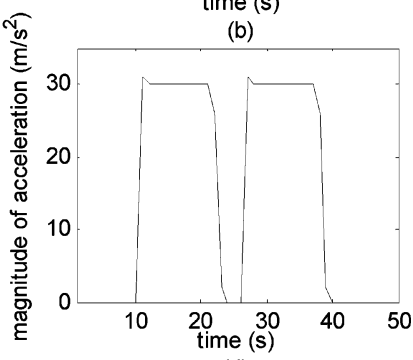

(d)
Fig. 1. State to be estimated in the orthogonal $\xi-\eta$ (Cartesian) coordinates. (a) Position trajectory in $\xi-\eta$ coordinates. (b) and (c) Velocity and acceleration trajectories in $\xi$ (solid line) and $\eta$ (dashed line). (d) Acceleration magnitude.
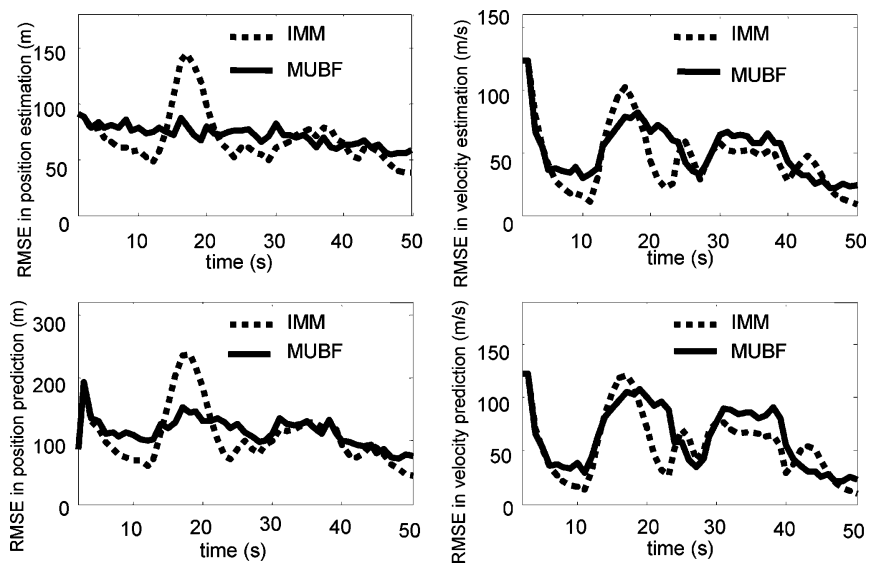

Fig. 2. RMSE of position estimation and prediction.

IMM, the MUBF supposes that the target maneuver is unknown a priori and treats it completely as GDI. In maneuvering target tracking, the tracking loss comes mainly from the abrupt maneuvering actions, which lead to significant uncertainties in target position. Therefore, the peak root-mean-square-error (RMSE) in the position prediction and estimation are the most important indexes for filter performance evaluation. Fig. 2 shows the RMSE of the IMM and MUBF via 1000 Monte Carlo simulations. Compared with the IMM, the MUBF reduces greatly the peak RMSE in target position estimation and prediction due to abrupt maneuvers.

The simulation PC is a HP nc4010. The programming language is Matlab 6.5 with LMI solver "mincx.m". The average running time for each simulation is $6.06 \mathrm{~ms}$ in MUBF and 6.70 $\mathrm{ms}$ in IMM. The $10 \%$ computation burden is saved.

\section{CONCLUSION}

This letter proposed a new discrete-time linear stochastic system model with the statistically-constrained DI, to which the existing DI-specific filters cannot be applied. Through constructing and minimizing the upper bounds of covariance 
matrices of state prediction, filtering residual, and state estimate, the MUBF was presented with adaptive filter parameters. The simulation of maneuvering target tracking showed that MUBF can significantly decrease the peak error in position, compared with the well-known IMM method.

\section{APPENDIX}

\section{PROOF OF THEOREM 3.1}

Existence of $U B F$ : It is needed to testify the existence of (6) and (8) because the third condition (7) of Theorem 3.1 is loose. As the first condition of Theorem 3.1, $P_{0 \mid 0}^{*} \geq P_{0 \mid 0}$ is guaranteed. Using mathematical induction, we assume $P_{k \mid k}^{*} \geq$ $P_{k \mid k}$ and testify (6) and (8). Substituting (13) and (16) into (20), there is

$$
H_{k+1}\left[P_{k+1 \mid k}^{*}-P_{k+1 \mid k}\right] H_{k+1}^{T} \geq 0 .
$$

Left multiply $H_{k+1}^{T}$ and right multiply $H_{k+1}$ in both sides of (A1), and there is

$$
H_{k+1}^{T} H_{k+1}\left[P_{k+1 \mid k}^{*}-P_{k+1 \mid k}\right] H_{k+1}^{T} H_{k+1} \geq 0 .
$$

Because the rank of $H_{k+1}^{T} H_{k+1}$ equals to that of $H_{k+1}$, and the rank is $n$ according to the second condition of Theorem 3.1, it is concluded that $H_{k+1}^{T} H_{k+1}$ is of full rank. Left and right multiply in both sides of (A.2), and we obtain (6). Substituting (6) into (17) leads to

$$
\begin{array}{r}
P_{k+1 \mid k+1}^{*} \geq\left(I-K_{k+1} H_{k+1}\right) P_{k+1 \mid k}\left(I-K_{k+1} H_{k+1}\right)^{T} \\
+K_{k+1} R_{k+1} K_{k+1}^{T}
\end{array}
$$

and (8) is obtained from (A3) and (14).

Optimal Filter Parameters: According to Definition 3.1, the set $\left\{\alpha_{k} \mid V_{k+1}^{*} \geq V_{k+1}\right\}$ will not be empty if a UBF exists. For any $\alpha_{1 k} \in \Lambda_{k}$, if $\alpha_{1 k} \leq \alpha_{2 k}$, then $\alpha_{2 k} \in \Lambda_{k}$ because $\left.V_{k+1}^{*}\right|_{\alpha_{1 k}} \leq\left. V_{k+1}^{*}\right|_{\alpha_{2 k}}$. Thus

$$
\begin{aligned}
\Lambda_{k} & =\left\{\alpha_{k} \mid \alpha_{k} \geq 1, V_{k+1}^{*} \geq V_{k+1}\right\} \\
& =\left\{\alpha_{k} \mid \alpha_{k} \geq 1\right\} \cap\left\{\alpha_{k} V_{k+1}^{*} \geq V_{k+1}\right\}
\end{aligned}
$$

is not empty, and hence, there exists $\alpha_{k}^{\text {Opt }}=$ : $\min \left\{\alpha_{k} \mid \alpha_{k} \in \Lambda_{k}\right\}$. It is only needed to testify that $\alpha_{k}^{O p t}$ and $K_{k+1}^{O p t}$ guarantee (21)-(23). From the definition of $\alpha_{k}^{O p t}, \alpha_{k} \geq \alpha_{k}^{O p t}$ exists to any $\alpha_{k} \in \Lambda_{k}$. Thus

$$
\begin{aligned}
& \Delta P_{k}=:\left.P_{k+1 \mid k}^{*}\right|_{\alpha_{k}}-\left.P_{k+1 \mid k}^{*}\right|_{\alpha_{k}^{O p t}} \\
& =\left(\alpha_{k}-\alpha_{k}^{O p t}\right) F_{k} P_{k \mid k}^{*} F_{k}^{T} \geq 0 \\
& \left.V_{k+1}^{*}\right|_{\alpha_{k}}-\left.V_{k+1}^{*}\right|_{\alpha_{k}^{o p t}} \\
& =\left(\alpha_{k}-\alpha_{k}^{O p t}\right) H_{k+1} F_{k} P_{k \mid k}^{*} F_{k}^{T} H_{k+1}^{T} \geq 0 \\
& P_{k+1 \mid k+1}^{*}\left|\alpha_{k}, K_{k+1}-P_{k+1 \mid k+1}^{*}\right|_{\alpha_{k}^{o p t}, K_{k+1}} \\
& =\left(I-K_{k+1} H_{k+1}\right) \Delta P_{k}\left(I-K_{k+1} H_{k+1}\right)^{T} \\
& \geq 0 \text {. }
\end{aligned}
$$

In deriving (A4) and (A6), the expressions of $P_{k+1 \mid k}^{*}$ in (15) and $P_{k+1 \mid k+1}^{*}$ in (17) are used, respectively. To obtain (A5), both expressions of $P_{k+1 \mid k}^{*}$ in (15) and $V_{k+1}^{*}$ in (16) are used.
From (16) and $R_{k+1}>0, V_{k+1}^{*} \geq R_{k+1}>0$ exists. Represent the symmetric and positive definite matrix $\left.V_{k+1}^{*}\right|_{\alpha_{k}^{O p t}}$ by $S_{k+1} S_{k+1}^{T}$, where $S_{k+1}$ is of full rank. Letting $D_{k+1}=$ : $\left.P_{k+1 \mid k}^{*}\right|_{\alpha_{k}^{O p t}} H_{k+1}^{T} S_{k+1}^{-T}$, there is

$$
\begin{aligned}
& \left.P_{k+1 \mid k+1}^{*}\right|_{\alpha_{k}^{O p t}, K_{k+1}} \\
= & \left.P_{k+1 \mid k}^{*}\right|_{\alpha_{k}^{O p t}}-D_{k+1} D_{k+1}^{T} \\
& +\left(K_{k+1} S_{k+1}-D_{k+1}\right)\left(K_{k+1} S_{k+1}-D_{k+1}\right)^{T} \\
\geq & \left.P_{k+1 \mid k}^{*}\right|_{\alpha_{k}^{O p t}}-D_{k+1} D_{k+1}^{T}=\left.P_{k+1 \mid k+1}^{*}\right|_{\alpha_{k}^{O p t}, K_{k+1}^{O p t}}
\end{aligned}
$$

where

$$
K_{k+1}^{O p t}=D_{k+1} S_{k+1}^{-1}=P_{k+1 \mid k}^{*} H_{k+1}^{T} V_{k+1}^{*-1} .
$$

With the fact that $\left(K_{k+1} S_{k+1}-D_{k+1}\right)$ $\left(K_{k+1} S_{k+1}-D_{k+1}\right)^{T} \geq 0$, the inequality (A7) is obtained. Thus, $\left.P_{k+1 \mid k+1}^{*}\right|_{\alpha_{k}^{O p t}, K_{k+1}}$ reaches its minimum (optimal) value if and only if (A8) is satisfied. As shown above, a UBF exists and $\alpha_{k}^{\text {Opt }} \in \Lambda_{k}$. Thus, (6)-(8) are obtained and (21)-(23) are further obtained based on (6)-(8) and (A4)-(A7).

\section{REFERENCES}

[1] R. K. Mehra, "Approaches to adaptive filtering," IEEE Trans. Autom. Control, vol. AC-17, no. 5, pp. 693-698, Dec. 1972.

[2] K. A. Myers and B. D. Tapley, "Adaptive sequential estimation with unknown noise statistics," IEEE Trans. Autom. Control, vol. AC-21, no. 4, pp. 520-523, Aug. 1976.

[3] Y. Liang, D. X. An, D. H. Zhou, and Q. Pan, "A finite horizon adaptive Kalman filter for linear systems with unknown disturbances," Signal Process., Elsevier, vol. 84, no. 11, pp. 2175-2194, 2004.

[4] Y. Liang, D. X. An, D. H. Zhou, and Q. Pan, "Estimation of timevarying time delay and parameters of a class of jump Markov nonlinear stochastic systems," Comput. Chem. Eng., Elsevier, vol. 27, no. 12, pp. 1761-1778, 2003.

[5] P. L. Bogler, "Tracking a maneuvering target using input estimation," IEEE Trans. Aerosp. Elect. Syst., vol. 23, no. 3, pp. 298-310, Jul. 1987.

[6] H. Lee and M. J. Tahk, "Generalized input-estimation technique for tracking maneuvering targets," IEEE Trans. Aerosp. Elect. Syst., vol. 35, no. 4, pp. 1388-1402, Oct. 1999.

[7] M. Darouach and M. Zasadzinski, "Unbiased minimum variance estimation for systems with unknown exogenous inputs," Automatica, vol. 33, no. 4, pp. 717-719, Apr. 1997.

[8] L. E. Ghaoui and G. Calafiore, "Robust filtering for discrete-time systems with bounded noise and parametric uncertainty," IEEE Trans. Autom. Control, vol. 46, no. 7, pp. 1084-1089, Jul. 2001.

[9] E. Mazor, A. Averbuch, Y. Bar-Shalom, and J. Dayan, "Interacting multiple model methods in multiple-multiple tracking: A survey," IEEE Trans. Aerosp. Elect. Syst., vol. 34, no. 1, pp. 103-122, Feb. 1998.

[10] B. Chen, "Interacting multiple model fixed-lag smoothing algorithm for Markovian switching systems," IEEE Trans. Aerosp. Elect. Syst., vol. 36, no. 1, pp. 243-250, Feb. 2000.

[11] Z. Wang, J. Lam, and X. Liu, "Robust filtering for discrete-time Markovian jump delay systems," IEEE Signal Process. Lett., vol. 11, no. 8, pp. 659-662, Aug. 2004

[12] L. A. Johnston and V. Krishnamurthy, "An improvement to the interacting multiple model (IMM) algorithm," IEEE Trans. Signal Process., vol. 49, no. 12, pp. 2909-2923, Dec. 2001.

[13] S. K. Katsikas, S. D. Likothanassis, G. N. Beligiannis, K. G. Berkeris, and D. A. Fotakis, "Genetically determined variable structure multiple model estimation," IEEE Trans. Signal Process., vol. 49, no. 10, pp. 2253-2261, Oct. 2001

[14] G. Tadmor and L. Mirkin, "H $\infty$ control and estimation with previewPart II: Fixed-size are solutions in discrete time," IEEE Trans. Autom. Control, vol. 50, no. 1, pp. 29-40, Jan. 2005. 